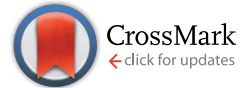

Cite this: J. Mater. Chem. C, 2014, 2, 6695

Received 18th April 2014 Accepted 29th May 2014

DOI: $10.1039 / \mathrm{c} 4 \mathrm{tc0} 0785 \mathrm{a}$

www.rsc.org/MaterialsC

\title{
Chiral-nematic liquid crystals as one dimensional photonic materials in optical sensors
}

\author{
D. J. Mulder, ${ }^{a}$ A. P. H. J. Schenning ${ }^{\star a}$ and C. W. M. Bastiaansen ${ }^{\star a b}$
}

Current developments in the field of thermotropic chiral-nematic liquid crystals as sensors are discussed. These one dimensional photonic materials are based on low molecular weight liquid crystals and chiralnematic polymeric networks. For both low molecular weight LCs and polymer networks, real-time and time integrating sensors have been realized. The response mechanism is either based on a change of helical twisting power of the dopant upon exposure to an analyte, or due to physical swelling, with a change of order in the liquid crystalline phase upon uptake of the analyte, causing the pitch to change. Sensors that respond to organic and water vapour, amines, water $\mathrm{CO}_{2}, \mathrm{O}_{2}$, metal ions, $\mathrm{pH}$, strain and temperature have been reported.

\section{Introduction}

Stimuli-responsive photonic crystals have received a lot of interest in the last few decades for possible applications in the field of optical sensors. ${ }^{1}$ The periodic alternation of the refractive index in these photonic materials leads to reflection of light which can be tuned by external stimuli. So far a number of fabrication methods have been reported to prepare responsive one, two and three dimensional (1D, 2D and 3D) photonic crystals. ${ }^{1}$ An appealing processing method is based on the selfassembly of liquid crystals. In a simple processing step, such as spin-coating, 1D photonic crystals can be prepared using socalled chiral nematic liquid crystals.

The liquid crystalline phase can be considered as a state of matter between the liquid- and the solid phase. Thermotropic liquid crystals are anisotropic partially ordered fluids which can exhibit different mesophases. The nematic phase has the lowest order of all mesophases and exhibits only directional order of the rod-like molecules. This directional order is described by the director $\mathbf{n}$. When a small amount of a chiral compound (dopant) is added, helical twisting of the molecular director $\mathbf{n}$ can be induced leading to a chiral nematic or a cholesteric liquid crystalline (CLC) phase (Fig. 1). The ability of a chiral dopant to induce a twist in the nematic phase is quantified by the helical twisting power (HTP). If the HTP of a chiral dopant in a certain mixture is known, the pitch $P$, the length of the helical axis over a $360^{\circ}$ rotation of the rod-like molecules, is calculated by $P=1 /(\beta \times$ ee $\times[C])$, where $\beta$ is the HTP, ee is the enantiomeric excess, and $[\mathrm{C}]$ the concentration of the chiral dopant.

${ }^{a}$ Laboratory of Functional Organic Materials and Devices, Eindhoven University of Technology, P. O. Box 513, 5600, The Netherlands. E-mail: a.p.h.j.schenning@tue. nl; c.m.w.bastiaansen@tue.nl

${ }^{b}$ School of Engineering and Materials Science, Queen Mary University of London, Mile End Road, London, E1 4NS, UK
Often, the cholesteric structure is drawn as a stack of layers of molecules with an orientational order, rotating each layer with a constant angle with respect to its neighbouring layer (Fig. 1). This very schematic representation of layers in a chiral-nematic structure has a limited physical reality. ${ }^{1}$ Each layer acts as a uniaxial anisotropic plane with the slow (extraordinary) axis parallel to the rod-like molecules and in most cases the fast (ordinary) axis perpendicular to the molecules. Throughout this 1D photonic material the two axes twist continuously through the layer. When light travels through the medium in the Bragg regime, incident light will be reflected (Fig. 1).

The colour of the reflected light depends on the helical pitch $P$ in following way: $\lambda_{\mathrm{b}}=\bar{n} \times P \times \cos \Theta$, where $\lambda_{\mathrm{b}}$ is the Bragg reflection wavelength (or selective reflection band (SRB)), $\bar{n}$ is the average refractive index $\left(\bar{n}=\left(n_{\mathrm{o}}+n_{\mathrm{e}}\right) / 2\right)$, where $n_{\mathrm{o}}$ and $n_{\mathrm{e}}$ are

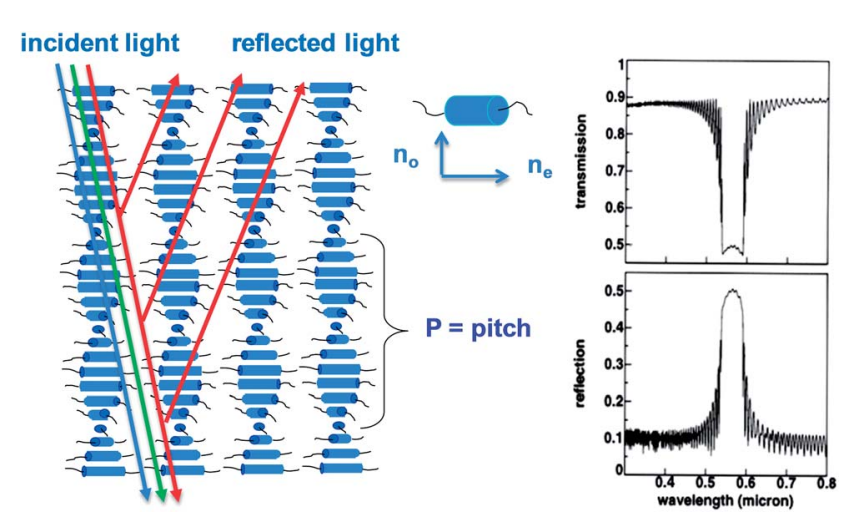

Fig. 1 Left hand side, the reflection of light by a chiral nematic liquid crystal. The material reflects only circular polarized light with the same pitch and handedness as the helical pitch and handedness of its helical superstructure. The other handedness is transmitted through the material. Right hand side, typical transmission and reflection spectra of a chiral nematic reflector. 
the ordinary and the extraordinary refractive indices, respectively, $P$ is the helical pitch and $\Theta$ the incidence angle of the light (Fig. 1). When the pitch length is in the regime of the visible light, the system will reflect visible light.

With the use of reactive mesogens, responsive chiral nematic polymer networks can be prepared with good mechanical strength. $^{2}$ Reactive mesogens can, for example, be provided with (meth-)acrylate groups and, in the presence of a photoinitiator, be photo-polymerized using UV-irradiation. In these materials the helical structure and the helical rotation are frozen in the polymeric network.

Cholesteric liquid crystals are appealing materials for use as optical sensors, due to their ability to form a one dimensional photonic structure by self-assembly. Cholesteric liquid crystals exhibit a wavelength and polarization selective reflection due to their periodic helical structure. ${ }^{2}$ The length of this helical pitch can be influenced by various external stimuli resulting in a change of the selective reflection wavelength (Fig. 2).

This makes it possible to use the cholesteric liquid crystals as optical sensors where changes can be observed with the naked eye. The stimulus, i.e., the analyte can react with the chiral dopant thereby changing its HTP and therefore the reflection colour of the system. The reflection colour can also change by swelling/shrinkage or a loss of the order in the LC mixture due to adsorption of the analyte by the photonic material.

The aim of this review is to present the recent developments in the field of thermotropic cholesteric low molecular weight liquid crystals (CLCs) and polymeric CLC networks as 1D photonic materials in optical sensors. Over the last few decades, responsive materials based on CLCs have also drawn their interest in, for instance, displays applications. In particular, the dynamic reflection colour change of CLCs to temperature, light and electrical stimuli has been explored. The recent developments in this field are beyond the scope of this article and have been presented in an excellent review by White et al. ${ }^{3}$ For the use of other liquid crystalline phases, lyotropic systems $^{4-7}$ and photonic crystals based on, for instance, 3D photonic materials as optical sensors the reader is referred to recent review papers. ${ }^{1,8-10}$

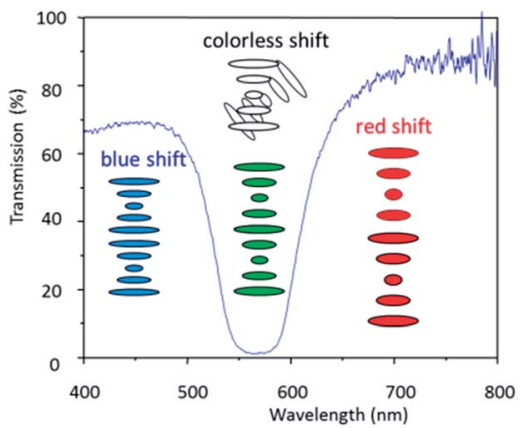

Fig. 2 Possible changes of the reflection band of a CLC polymer sensor upon responding to an analyte. Shrinkage of the polymer network leads to a blue shift, expansion to a red shift while a loss of molecular order leads to a disappearance of the green reflection band.

\section{Sensors based on low molecular weight CLC mixtures}

\section{Organic vapour sensors}

CLCs can act as optical vapour sensors by adsorption of solvent vapour resulting in a change in the pitch. The change in the pitch can be explained by the change in the twisting angle between the molecules or stretching the molecule along the helical main director.

In 1994 Dickert et al. investigated CLC sensors that are capable of sensing various organic vapour molecules (Fig. 3). ${ }^{\mathbf{1 1}}$ The CLC mixture consisted of cholesteryl chloride (CC), cholesteryl oleylcarbonate (CO), and cholesteryl nonaoate (CN). Upon exposure to these vapours a red-shift is observed. This shift is caused by the penetration of the solvent molecules into the film, leading to a distortion of the anisotropic phase resulting in a swelling of the helical pitch. The authors pointed out that in order to obtain reliable results from a CLC sensor environmental parameters such as temperature or relative humidity have to be minimized. The use of intelligent data interpretation was suggested to filter out the cross sensitivity signals caused by these environmental parameters in which the temperature and relative humidity are considered as additional analytes. With this intelligent data interpretation method, a linear response and a low detection limit $(<10 \mathrm{ppm})$ were obtained. The selectivity could be tuned by using different ratios of the LCs giving different responses to different solvents.

Winterbottom et al. explored a similar system involving a binary CLC mixture. ${ }^{12}$ The sensor was exposed to ethanol,
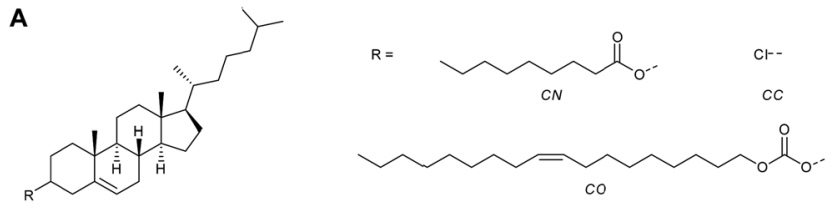

B

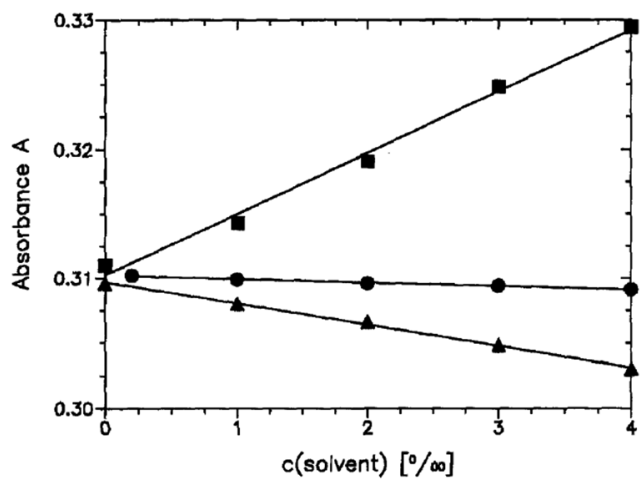

Fig. 3 (A) Chemical structures of cholesterol derivatives used in the CLC sensors to sense organic solvent vapour in the work of Dickert et al. and (B) the sensor characteristics of a cholesteric liquid crystal absorbance $A$ at $398 \mathrm{~nm}$ versus solvent vapour concentration in air at $20{ }^{\circ} \mathrm{C}$ (CC : CN : CO = 3:16:12-weight ratio) $(\boldsymbol{0}=$ methanol, $\mathbf{\square}=$ chloroform, and $\boldsymbol{\Delta}=$ tetrahydrofuran). Adapted from Dickert et al. ${ }^{11}$ Reproduced with kind permission from Springer Science and Business Media. 
acetone, benzene, pyridine, and hexane vapour and a reversible red-shift response of the SRB was observed within 3 minutes of exposure. Unfortunately, the stability of the CLC films was poor. Crystallization of the components was observed 200 minutes after preparation of the films. The authors stabilized the CLC by immobilizing it in a polymer matrix. As a polymer binder, 0.1$0.5 \%$ PVC or silicon rubber was used. The lifetime of the sensor was increased and no crystallization was observed under ambient conditions. The authors tested the polymer stabilized CLC sensors by exposing them to acetone vapour and a change in the SRB of the liquid crystal was observed. The response time of the polymer stabilized CLCs was found to be dependent on the concentration of the polymer.

Mujahid et al. prepared a CLC sensor based on chiral biphenyls (Licritherm TM 1013, Merck AG). ${ }^{13}$ CLC films were prepared and exposed to ethanol, tetrahydrofuran (THF), chloroform, and tetrachloroethylene vapour and a blue-shift of the SRB was observed. A linear correlation was found between the shift of the absorbance $(\Delta A)$ and the molecular mass of the solvents examined (Fig. 4A). The authors also investigated the lifetime of the CLC sensor films. They prepared a cross-linked polystyrene matrix in which the CLC was dispersed. Three polymer films were prepared with $5 \%, 10 \%$, and $20 \%$ CLC. The $5 \%$ CLC film showed clear discrimination between THF and ethanol and the selectivity for the polymer stabilized CLC sensors was higher than that for the pure CLC mixture. The response of the stabilized film shows linear behaviour as a

A
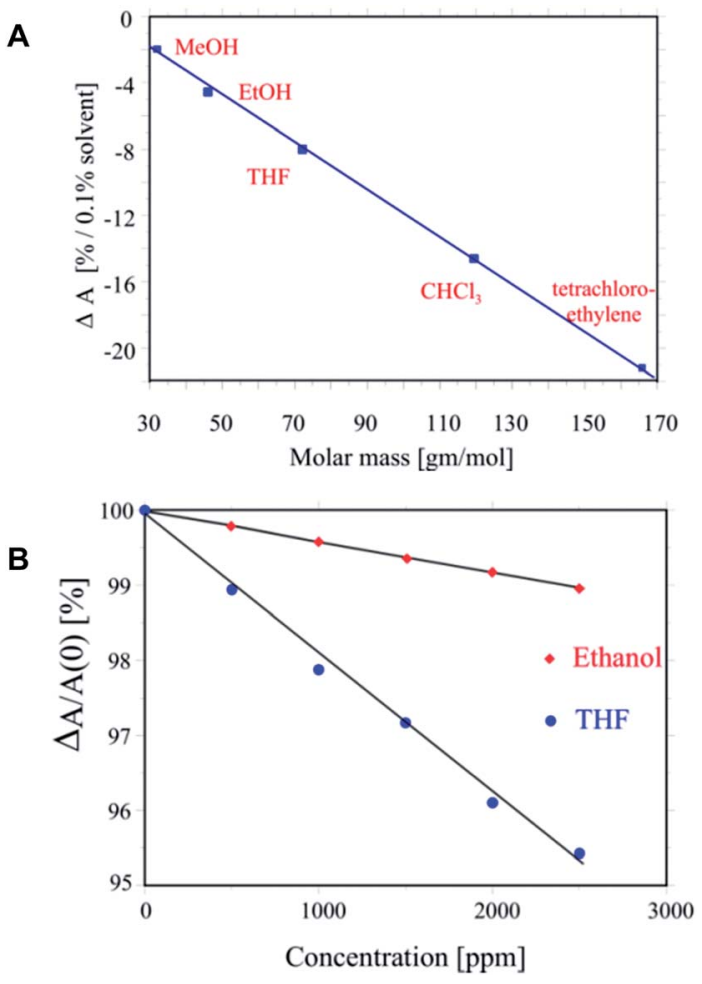

Fig. 4 (A) Optical sensor response of CLCs towards molar masses of different analyte vapours. (B) Optical response of CLCs embedded in the imprinted polymer for THF and EtOH showing enhanced selectivity for imprinted THF than EtOH. Adapted from Mujahid et al. ${ }^{13}$ function of the concentration of organic vapour present (Fig. 4B). Furthermore, films based on 5\% CLC and polystyrene showed good mechanical stability.

Chang et al. studied a CLC organic vapour sensor based on the commercial nematic LC mixture E7 (Merck). ${ }^{\mathbf{1 4}}$ Two chiral dopants were used to induce the cholesteric twist, S1011 (17 wt\%, Merck) and DBD (2 wt\%, WeiFlex) (Fig. 5A). Films with a thickness of $20 \mu \mathrm{m}$ were prepared exhibiting a SRB that redshifted upon exposure to acetone and toluene vapours. The initial colour of the film was cyan and the final state was transparent. Polarized light microscopy and UV-Vis spectroscopy revealed that when solvent vapour diffused in the CLC, a phase transition occurred to the isotropic phase resulting in a transparent film. Acetone showed a stronger and faster response than toluene due to a favourable interaction with the cyano groups in the CLC. Furthermore, the authors showed that the diffusion occurred by using a Fickian type II diffusion model by fitting the time dependent SRB shift.

In a later paper the authors demonstrated that by using carbon nanotubes (CNTs) the dynamic range of the sensor can be enhanced by combining the optical readout with an electric resistance measurement. ${ }^{15}$

A hybrid CLC sensor containing CNTs (1.5\%) was prepared and upon exposure to acetone a clear red-shift was observed up

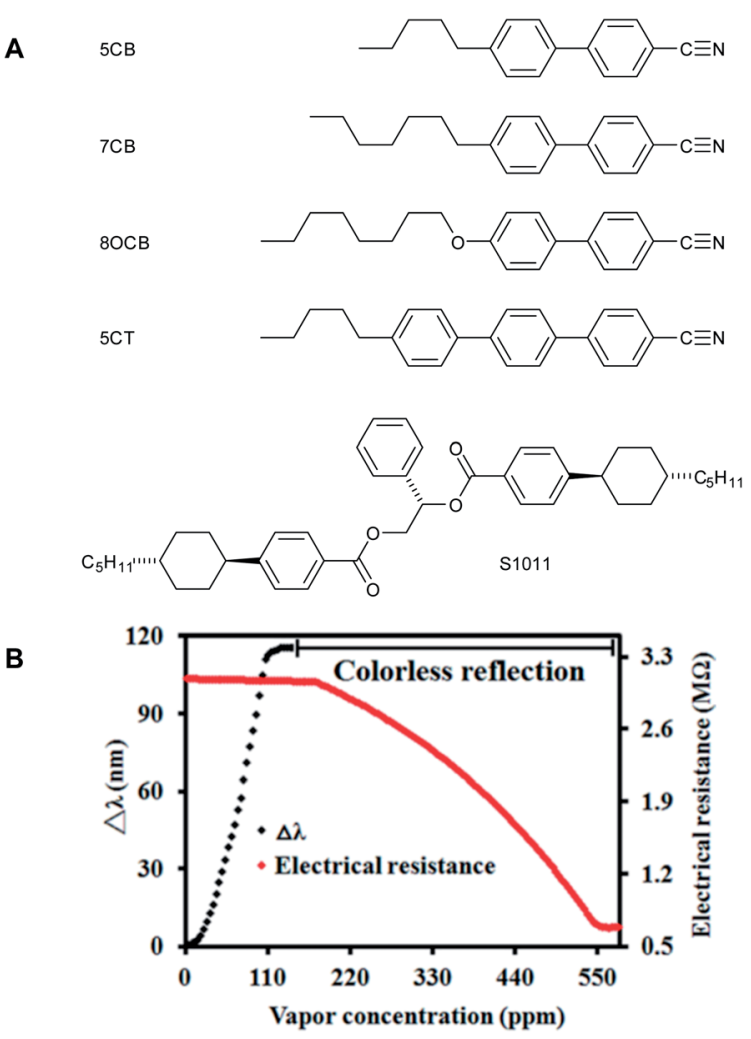

Fig. 5 Structures of the components present in the nematic mixture E7 (5CB, 7CB, 8OCB, and 5CT) and the chiral dopant (S1011) used in the work of Chang et al. ${ }^{14}$ and the change in reflection wavelength and electrical resistance as a function of vapour concentration. Reprinted with permission from C.-K. Chang, S.-W. Chiu, H.-L. Kuo, and K.-T. Tang, Appl. Phys. Lett., 2012, 100, 043501. Copyright 2014, AIP Publishing LLC. 
to a vapour concentration of $140 \mathrm{ppm}$. Acetone diffuses into the CLC film, causing a swelling of the pitch and ultimately a total disruption of the liquid crystal assembly. Polarized light microscopy revealed that the Grand Jean texture of the CLC phase transforms to a multi-domain texture in a branching motion. The authors explain that during this transformation the CNTs branch out and form a conducting network, resulting in a significant change in conductive properties. Initially the film shows a high electric resistance of 3.09 $\mathrm{M} \Omega$. During the branching the resistance decreases to $0.72 \mathrm{M} \Omega$. This branching motion appears in the range of 160-550 ppm giving the sensor a high sensitivity in this range (Fig. 5B).

\section{Carbon dioxide sensor}

With the use of responsive dopants, a selective and large response of the helical pitch to analytes can be achieved in CLC sensors. The concept is based on the change of HTP of the chiral dopant when the dopant reacts selectively with the analyte molecules. ${ }^{16,17}$ In the research of Han et al. two sensors were investigated, one that can monitor $\mathrm{CO}_{2}$ real time (reversible) and another that can record oxygen exposure over time (irreversible). The carbon dioxide sensor was prepared by using an optically pure diamine with a TADDOL derivative (Fig. 6A) as a chiral dopant in the nematic LC mixture E7. ${ }^{18}$ The diamines react with $\mathrm{CO}_{2}$ forming carbamates (Fig. 6B).

The pure chiral diamine has a low HTP in E7. Therefore, the authors prepared 1:1 hydrogen bonded complexes of the diamine with a TADDOL derivative (Fig. 7a) which has a high twisting power $\left(\beta=+250 \mu \mathrm{m}^{-1}\right)$. Films containing the $1.6 \%$ diamine-TADDOL complex in E7 were prepared. The films prepared with $(R, R)$-amine-TADDOL exhibit a SRB at $637 \mathrm{~nm}$, which corresponds to a red colour reflection. When the films were exposed to $\mathrm{CO}_{2}$ a blue-shift occurred, yielding a green film $(\mathrm{SRB}=495 \mathrm{~nm})$ that is clearly visible in Fig. 6C. This indicated a shorter pitch due to a higher HTP of the dopant. The response of the CLC sensor was fast: the colour of the film shifted within

A
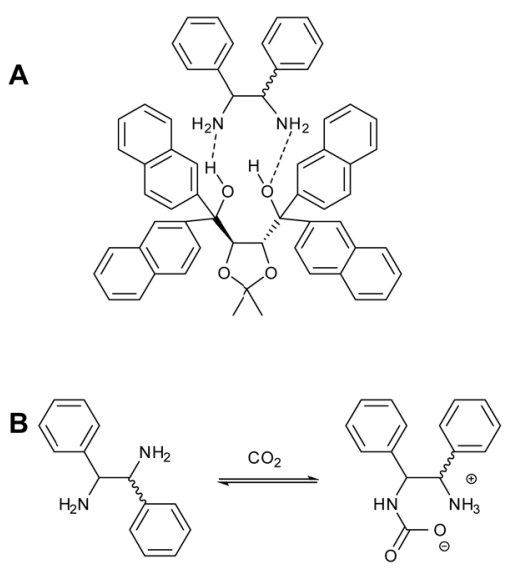

Fig. 6 (A) Structure of $1: 1$ amine-(R,R)-TADDOL derivative complex, (B) the carbamate formation of the amine compound, and (C) images of the CLC film before (top) and after (bottom) exposure to $\mathrm{CO}_{2}$ for $1 \mathrm{~h}$. Adapted with permission from Saha et al. ${ }^{17}$ Reproduced with permission of The Royal Society of Chemistry.
A

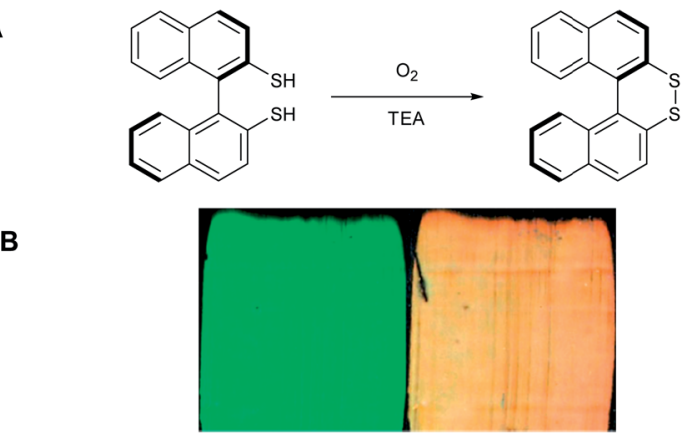

Fig. 7 (A) Oxidation of the binaphtalene dithiol to disulfide bridged species by $\mathrm{O}_{2} . \beta=13 \mu \mathrm{m}^{-1}$ for the dithiol species, and $\beta=65 \mu \mathrm{m}^{-1}$ for the disulfide bridged species, (B) images of the CLC film before (left) and after (right) exposure to air for $1 \mathrm{~h}$ in the presence of triethylamine. Adapted with permission from Y. Han, K. Pacheco, C. W. M. Bastiaansen, D. J. Broer, and R. P. Sijbesma. ${ }^{16}$ Copyright 2014 American Chemical Society.

10 seconds. Kinetic studies showed that the response of the sensor was not diffusion controlled but predominantly chemical reaction controlled, following a rate-limiting pseudo-firstorder reaction.

\section{Oxygen and amine sensor}

An oxygen sensor based on the oxidation of thiols by molecular oxygen to disulfides was also prepared. ${ }^{17} \mathrm{~A}$ chiral binaphtalene dithiol was used as the dopant which in the presence of a small amount of catalyst (e.g. triethylamine (TEA)) can be oxidized to the disulfide dopant. The open dithiol derivative exhibits a lower HTP $\left(\beta=13 \mu \mathrm{m}^{-1}\right)$ than the disulfide bridged derivative $\left(\beta=65 \mu \mathrm{m}^{-1}\right)$. Contrarily, due to the low solubility and precipitation of the disulfide bridged species the SRB changed in the opposite direction (Fig. 7).

The initial green colour $(\mathrm{SRB}=542 \mathrm{~nm})$ changed to orange $(\mathrm{SRB}=600 \mathrm{~nm})$. Control experiments showed that when the film was exposed to air without TEA, or TEA vapour in a nitrogen atmosphere, no colour change occurred. This latest result reveals the potential to use the sensor for volatile amines in an oxygen rich atmosphere which could be interesting for the detection of volatile amines that are produced by the bacterial decomposition of fish. This detection can be of interest in, for instance, food packaging. ${ }^{19}$

\section{Humidity sensor}

Saha et al. prepared a (irreversible) humidity sensor based on bis-(binaphtylenedioxy)silane (Fig. 8) as a reactive chiral dopant in E7. ${ }^{17}$ This chiral compound is very sensitive to hydrolysis. Upon exposure to humid air the silane compound hydrolyses, yielding two binaphthol compounds.

The silane compound and the hydrolysed products have a similar helical twisting power, $-34.9 \mu \mathrm{m}^{-1}$ versus $-31.7 \mu \mathrm{m}^{-1}$, respectively. Nevertheless, a significant change of the reflection band was observed when a film containing $10.1 \%$ silane dopant in E7 was exposed to humid air. 


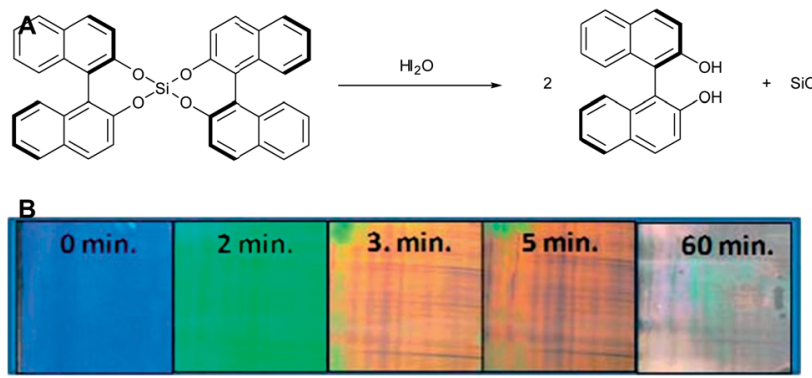

Fig. 8 (A) The reaction of bis-[binaphthylen-(2.2')-dioxy]-silan with water to form $(R)-(+)-1,1^{\prime}-\mathrm{bi}\left(2-\right.$ naphthol) and $\mathrm{SiO}_{2}$ and $(\mathrm{B})$ photographs indicating colour changes upon exposure to air (relative humidity of $75 \%$ ) of a CLC film of E7 containing $10.1 \%$ of bis-[binaphthylen-(2.2')dioxy]-silan. Saha et al. ${ }^{17}$ Reproduced with permission of The Royal Society of Chemistry.

The SRB of the initial blue film red-shifted to orange-red within a few minutes and the film turned colourless within an hour. The binaphthol products are poorly soluble in the E7 matrix, resulting in precipitation. The rate of the SRB-shift was found to be sensitive to the relative humidity. However, regardless of the relative humidity, a colourless film was obtained after long exposure even to a low relative humidity, indicating good time-analyte integrating properties. The authors showed that the rate at which the SRB-shift occurred was not dependent on the diffusion rate of the analyte since the rate of the SRB-shift was the same for samples with different thicknesses.

\section{Sensors based on polymeric CLC networks}

\section{pH and amino acid sensors}

In 2002 the first CLC polymer $\mathrm{pH}$ sensor was reported by Shibaev et al. ${ }^{20} \mathrm{~A}$ chiral polymer composite with a frozen cholesteric structure was prepared by the photo-polymerization of a hydrogen bonded mesogenic acrylate (AHA) in the presence of a cross-linker (DIAB), a hydrogen-bonded chiral dopant (MMA) and a non-reactive hydrogen-bonded mesogen (PCA) (see Fig. 9A). The authors prepared various mixtures of the compound with different compositions in which the photopolymerization was performed in the cholesteric phase. With a concentration of the chiral dopant between $12 \%$ and $23 \%$ the selective reflection band is in the visible spectral region. With high cross-link densities $(>35 \%)$ the long term preservation of the helical superstructure after cooling of the sample was realized. When the polymer film was exposed to basic water the hydrogen bonds were disrupted.

This deprotonation lead to the formation of hygroscopic carboxylate salts. The uptake of water by this salt caused the material to swell and the cholesteric pitch length, and thus the selective reflection band, to change. This effect results in a red shift of the position of the selective reflection band (Fig. 9B). In subsequent studies, a slightly altered system was explored in which the sensitivity towards basic amino acids was also considered. ${ }^{21-23}$ This was done by exposing 4 different types of samples to basic amino acid solutions. The first sample containing no hydrogen bonded moieties did not respond to the basic arginine solution. The second sample containing an inert chiral dopant (ZLI 4572) and a hydrogen bonded polymer matrix did respond very weakly. A shift of 15-20 nm of the SRB was observed. The third sample containing the hydrogen bonded dopant MAA and an inert polymer matrix, thus without AHA, did not change colour. The fourth sample containing the hydrogen bonded chiral dopant and the hydrogen bonded AHA showed a strong response to the amino acid solution.

Furthermore, an increase of AHA in the mixture leads to a more hydrophilic polymer film, a faster response, and a greater shift of the SRB. At high concentrations of AHA the quality and stability of the SRB was poor. The wet films did swell physically in thickness by a factor of 1.2-1.8.

A

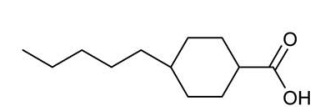

PCA<smiles>C=CC(=O)OCCCCCCOc1ccc(C)cc1</smiles>

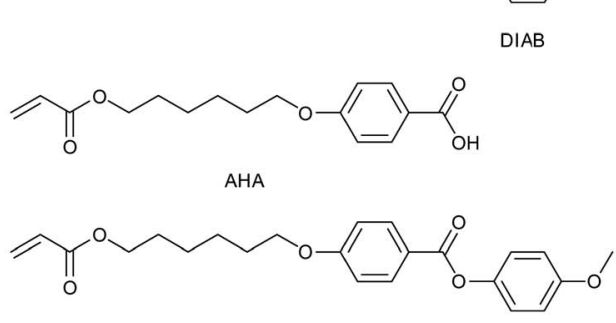

ABM<smiles>CC(CCC(=O)O)CC(=O)O</smiles>

MAA

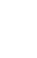<smiles>C=CC(=O)OCCCCCCOc1ccc(C(=O)Oc2ccc(OC(C)=O)cc2)cc1</smiles><smiles>N=C(N)NCCCC(N)C(=O)O</smiles>

ARG 
Later Chen et al. developed a hydrogen bonded CLC network with double sensing properties. The material is sensitive for both $\mathrm{pH}$ and temperature over a wide temperature range. ${ }^{24}$ But contrary to the chiral dopant used by Shibaev et al., this system contains a chiral dopant that only can act as a hydrogen bond acceptor (Fig. 10A). Furthermore, the system contains a nematic mixture of liquid crystalline acrylates and an inert chiral liquid crystalline acrylate. In a previous study it was shown that the HTP of hydrogen-bonded chiral dopant changes with increasing temperature. ${ }^{25}$ This effect was also observed for photo-polymerized samples. A change in SRB was observed from $570 \mathrm{~nm}$ (green/yellow) to $611 \mathrm{~nm}$ (yellow/red) when the polymer film was heated from $60{ }^{\circ} \mathrm{C}$ to $200{ }^{\circ} \mathrm{C}$. During heating the hydrogenbonds are weakened gradually over a wide temperature range. This colour change was reversible because when the sample was cooled to $60{ }^{\circ} \mathrm{C}$ again a green/yellow film was obtained.

In further studies, five samples were placed at different $\mathrm{pH}$ solutions and allowed to equilibrate for 5 days (Fig. 10B). The colour of the film changes above $\mathrm{pH} 7$ and at higher $\mathrm{pH}$ values the shift of the SRB is greater. The concentration of the hydrogen-bonded chiral dopant present in the polymer film influences the rate and the magnitude of the SRB shift (Fig. 10C). A higher concentration of the hydrogen bonded assembly leads to a greater shift.

Similar to the aforementioned investigation of Shibaev et al., the authors observed the disappearance of the signals in FTIR spectra corresponding to hydrogen bonded carboxylic acid and the appearance of the signals that indicate carboxylate salt formation. The breakage of the hydrogen bonds, and the formation of carboxylate groups, leads to a swelling of the helical pitch, and subsequently a red-shift of the selective reflection wavelength.

\section{Alcohol sensor}

An alcohol responsive sensor has been developed by Chang et $a .^{26,27} \mathrm{~A}$ nematic polymerizible LC mixture was used containing two mesogens with benzoic acid moieties (Fig. 11). Furthermore, the mixture contained a chiral moiety to induce the helical twist. A non-reactive nematic mesogen 5CB (see Fig. 5A) was added to the mixture. This non-reactive mesogen was subsequently removed after the photo-polymerization to obtain a porous CLC network. Interestingly, this porosity leads to a faster response of the sensor since the surface area through which the reagents diffuse increased. The sensor films were prepared by inkjet printing the cholesteric liquid crystal mixture on rubbed triacetyl cellulose (TAC) foil. After photopolymerization and the removal of the porogen, the films were activated in an alkaline solution. The hydrogen bonds between the benzoic acid moieties in the polymer film were broken and sodium salt was formed. These ionic carboxylate groups have different affinities for different alcohol-water mixtures.

The activated CLC network films were dried and subsequently exposed to solutions of $40 \%$ alcohol in water. A red-shift of the SRB was observed. This shift is caused by the swelling of the film due to the adsorption of the alcohol in the CLC network. A clear distinction could be made between methanol and ethanol. For ethanol-water mixtures the shift of the SRB

A
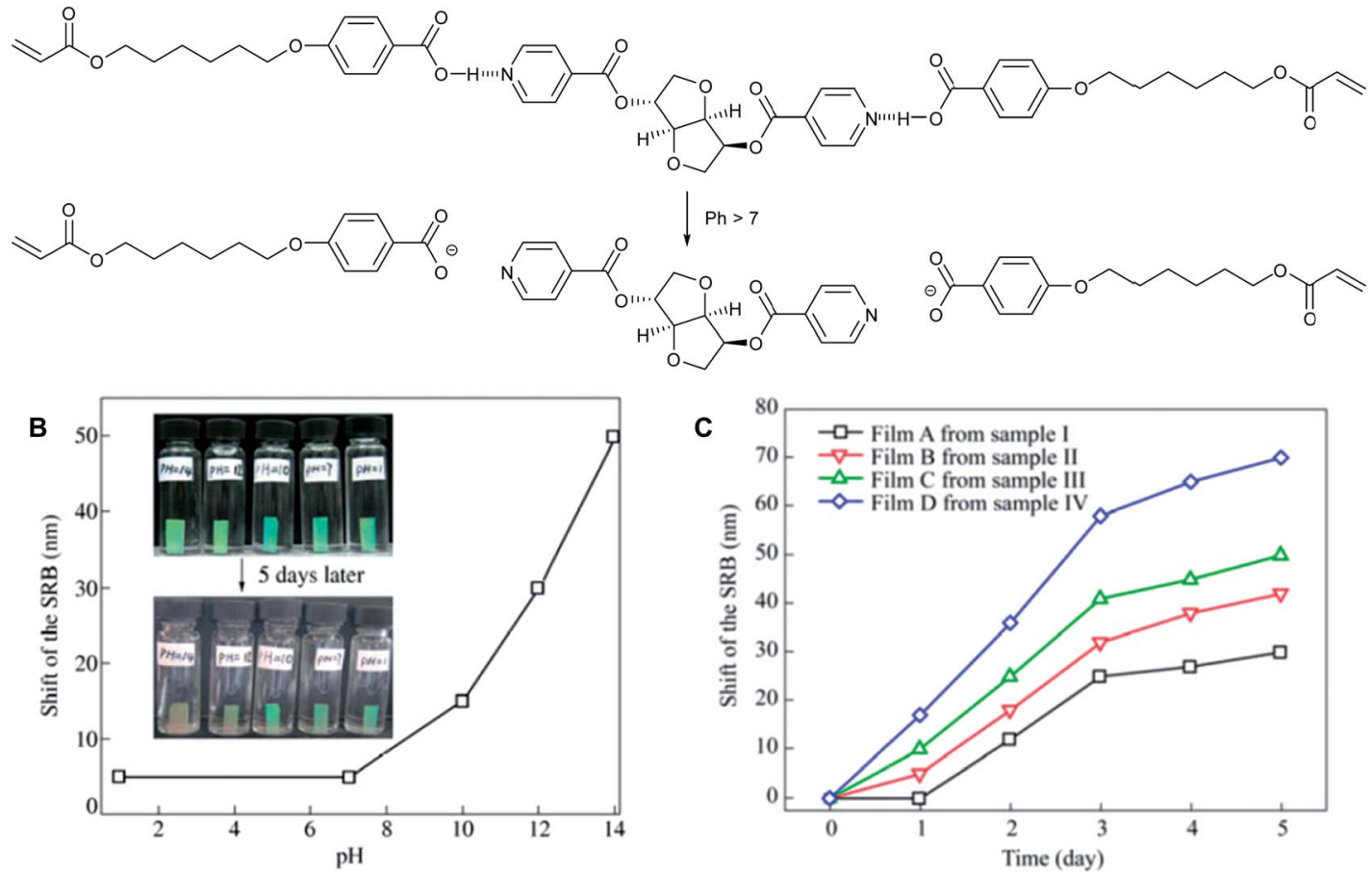

Fig. 10 (A) Schematic of breaking $\mathrm{H}$-bonds in the cholesteric polymer films, (B) shift of the SRB of the film A after treatment in aqueous solutions with different $\mathrm{pH}$ values for 5 days (the insets are the corresponding photos of the initiative films and the films after 5 days treatment), and (C) SRB shift of films as a function of treating time in aqueous solution with $\mathrm{pH}=12$ (bottom right). Adapted from Chen et al. ${ }^{24}$ With kind permission from Springer Science and Business Media. 
A

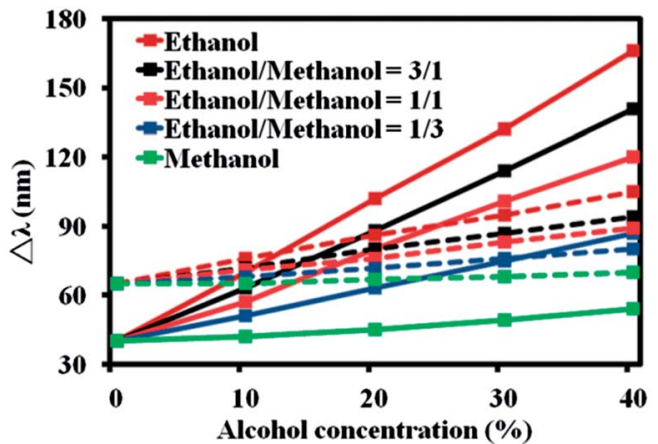

B

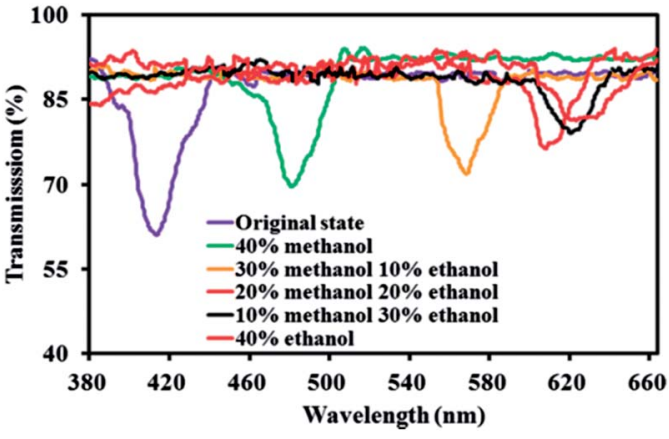

C

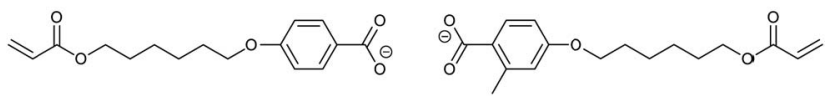

Fig. 11 (A) The solid and dashed lines represent changes in the transmission-valley wavelength of CLC films with and without porosity, respectively in alcohol solutions, with different alcohol concentrations and ratios of methanol/ethanol. (B) Optical transmission spectra of CLC in alcohol solutions with different ratios of methanol/ethanol. Reprinted (adapted) with permission from C.-K. Chang, C. W. M. Bastiaansen, D. J. Broer, and H.-L. Kuo, Macromolecules, 2012, 45, 4550-4555. Copyright 2014 American Chemical Society. (C) Chemical structures of the activated carboxylate moieties used in the alcohol sensor of Chang et al. 26,27

was larger than for methanol-water mixtures. In an experiment where the authors exposed the CLC networks to various ratios of methanol/ethanol they showed that it is possible to deduce the ratio from the shift of the $\operatorname{SRB}(\Delta \lambda$, Fig. 11A). The authors explained the difference in the response of methanol and ethanol by comparing the Hildebrand solubility parameters of the solvents with the ones of benzoic acid (binding moieties of the sensor interior). The Hildebrand solubility parameters are $29 \mathrm{MPa}^{0.5}$ and $26 \mathrm{MPa}^{0.5}$ for methanol and ethanol, respectively. The value of benzoic acid is $22 \mathrm{MPa}^{0.5}$. Therefore, the authors suggest that the affinity for ethanol is higher. Sensors that are able to discriminate between alcohol molecules are potentially interesting for counterfeited alcoholic beverages containing, for instance, toxic methanol.

\section{Metal ion sensor}

Stroganov et al. have fabricated a cation sensitive polymer stabilized CLC composite using crown ether moieties in the cholesteric LC polymer network (Fig. 12). ${ }^{28}$ Crown-ethers are well known to form host-guest complexes upon binding with metal ions in which the binding affinity of a certain metal ion is dependent on the size of the crown-ether and the ionic radius of the metal ion. The crown ether moiety used in this investigation is based on 18-crown-6, and the analytes that were investigated were barium and potassium. The composition exists partly from a cholesteric matrix of unreactive mesogens and mono- and diacrylates, yielding a stabilized CLC composite after photopolymerization. In this way a mechanically stable CLC film is obtained which still has a response to analytes (Fig. 12A).

Upon contact with a $0.01 \mathrm{M}$ (aq.) solution of barium perchlorate the selective light reflection shifted to shorter wavelengths, indicating a decrease of the pitch of the cholesteric helix. The authors address this observation to two possible origins. First, the microphase separation of the crown ether-metal complex induces additional twisting of the supramolecular helical pitch of the cholesteric matrix and secondly to a slight collapse of the polymer network caused by the attractive interaction between charged moieties in the crown ether-metal complex. The authors showed that the binding constant of the crown ether was higher for the bivalent barium ions than the monovalent potassium ions with a similar ion radius. The authors did not investigate the selectivity towards metal ions other than barium and potassium, nor the influence of the counter ions.

\section{Humidity sensor}

In an investigation by Herzer et al. an optical humidity sensor was developed. ${ }^{29}$ The sensor is based on swelling of the CLC polymer film caused by the absorption of water by the hygroscopic properties of the potassium carboxylate salts in the film. This swelling leads to an increase of the cholesteric pitch, causing the SRB to shift to red. The composition of this CLC polymer film exists of two compounds that can form hydrogenbonds (Fig. 13), a chemical cross-linker, a mono-functional mesogenic acrylate, and a chiral mesogenic acrylate to induce a cholesteric pitch. The mixture was applied on a rubbed polyimide coated glass substrate by bar-coating. Subsequently, the coating was polymerized by UV light. The concentration of the chiral compound was such that a film was obtained with a SRB at $\lambda=540 \mathrm{~nm}$ (green colour reflection). In order obtain the humidity sensing properties, the hydrogen-bonded benzoic acid moieties had to be converted to carboxylate groups. This was done by exposing the CLC network film to a $0.05 \mathrm{M} \mathrm{KOH}$ (aq.) solution. Upon exposure to the alkaline solution the film displayed a red-shift until it became colourless (SRB in infrared region). After drying the colour of the resulting carboxylate salt containing polymer film changed back to green, a SRB of $\lambda=$ $550 \mathrm{~nm}$ was obtained.

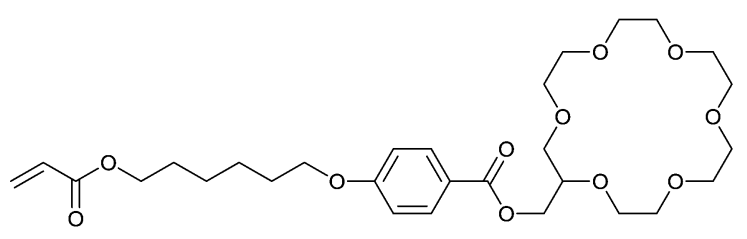

Fig. 12 The chemical structures of components in the stabilized CLC composite. Adapted from Stroganov et al. ${ }^{28}$ Copyright (c) 2012 WILEYVCH Verlag GmbH \& Co. KGaA, Weinheim. 
A

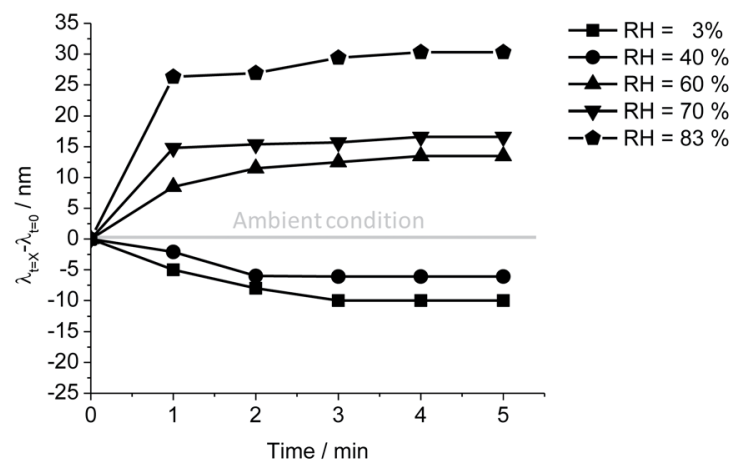

B

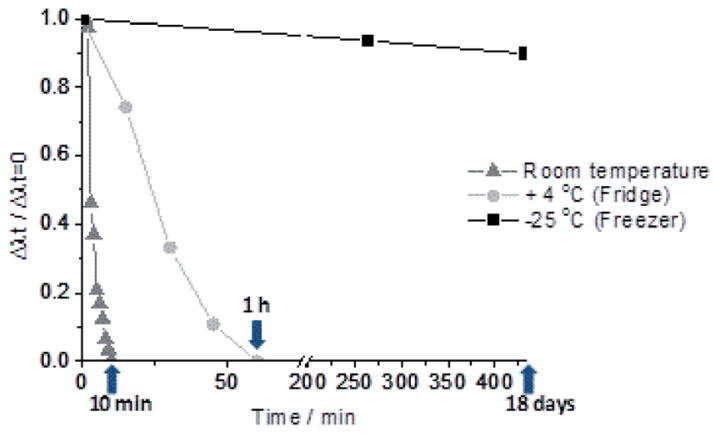

C

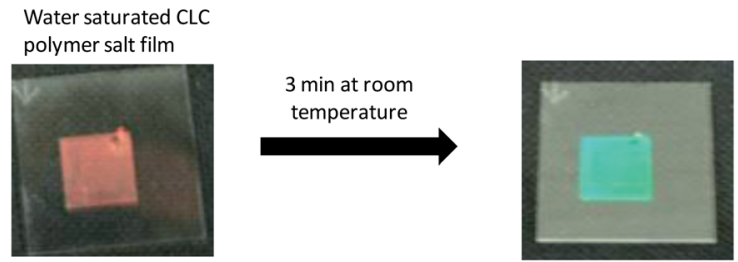

Fig. 13 (A) The relative shift of SRB to the original SRB (ambient condition) wavelength versus time is plotted for 3, 40, 60, 70 and $83 \%$ $\mathrm{RH}$. (B) Time response of the water saturated polymer salt film at room temperature, refrigerator and freezer temperatures: the $y$-axis signal $\Delta \lambda_{t} / \Delta \lambda_{t=0}$ is used as a measure for the response versus time at different temperatures. The lines added are a guide to the eye. (C) Photographs of the inkjet-printed water saturated CLC polymer salt film on polyimide coated glass in water (left red film) and after 3 minutes at room temperature (right green film). Adapted with permission from $\mathrm{N}$. Herzer, H. Guneysu, D. J. D. Davies, D. Yildirim, A. R. Vaccaro, D. J. Broer, C. W. M. Bastiaansen, and A. P. H. J. Schenning, J. Am. Chem. Soc., 2012, 134, 7608-11. Copyright (2012) American Chemical Society.

The CLC network films were exposed to different relative humidities (RH). If the sensor is exposed to lower $\mathrm{RH}$ than the ambient conditions a blue-shift is observed, and thus the cholesteric pitch shrinks due to the evaporation of water from the CLC network film (Fig. 13A). If the $\mathrm{RH}$ is higher than ambient conditions, a red-shift is observed. Water is adsorbed by the hygroscopic carboxylate moieties causing a swelling and increase of the cholesteric pitch.

In an additional experiment the processability of the material was investigated. The material was inkjet printed on rubbed polyimide coated glass and rubbed polymer foil. The samples were aligning well on both substrates. After the activation step a strong green reflection was obtained. The response of the printed samples was faster than the bar-coated films. The authors explained this effect due to larger defects present in the printed film resulting in a more porous system.

Furthermore, the authors found that when the polymer film was fully saturated with water it could be used as a temperature sensor (Fig. 13B and C). The water saturated CLC film was allowed to equilibrate at room temperature, $4{ }^{\circ} \mathrm{C}$, and $-24{ }^{\circ} \mathrm{C}$. At low temperatures (below the freezing point of water) the response of the film was extremely slow. Above the freezing point of water, the sensor responds in an irreversible fashion showing that it is possible to utilize this material as a temperature sensor.

\section{Time-temperature sensor}

With the same liquid crystal mixture as used by Herzer et al., but not treated with alkaline solution, Davies et al. prepared an optical time-temperature $(t-T)$ integrator. ${ }^{30}$ CLC network films were prepared either by a bar-coating process or inkjet printing. After photo-polymerization the films were mechanically embossed above the glass transition temperature $\left(T_{\mathrm{g}}=60^{\circ} \mathrm{C}\right)$ using a rigid sphere stamp (Fig. 14a).

The indention in the material was $5-10 \%$, resulting in a shift of the SRB of $30 \mathrm{~nm}$. The embossed samples were stable at room temperature. The rate of recovery becomes increasingly faster with increasing temperature (Fig. 14c). When the samples were heated to $40-50{ }^{\circ} \mathrm{C}$ the SRB changed back irreversibly to the initial state. The response of the sensor is based on the shape memory effect of the material. When the sample was heated above its $T_{\mathrm{g}}$ the embossed spot flattens and forms a smooth surface (Fig. 14b).

\section{Amine sensor}

Recently a printable hydrogen-bonded cholesteric liquid crystal (CLC) polymer film has been reported, that can be used as a sensor for detection of gaseous trimethylamine (TMA). ${ }^{31}$ In this optical sensor based on a similar chemical mixture as reported by Shibaev et al. the virgin CLC polymer network reflects green light. When exposed to TMA in water-saturated nitrogen gas results in a red reflecting film. Due to the hygroscopic nature of the polymer salt that is formed by TMA, water vapor which is present in the environment is absorbed by the films. This leads to swelling of the film, resulting in an increase in the pitch size and therefore a red shift of the reflection band (see Fig. 15). Interestingly, after exposure to ambient conditions, restoration of the green reflecting film takes place, showing that the sensor can be used multiple times. In a proof of principle experiment, it was also shown that these CLC films can be used as optical sensors to detect volatile amines that are produced by decaying fish.

\section{Strain sensor}

A flexible, strain sensor has recently demonstrated for monitoring uniaxial deformations in oriented polymer films (Fig. 16). ${ }^{32}$ This sensor consisted of a cross-linked cholesteric liquid crystal layer which was spray-coated on a polyamide substrate showing an orange reflection band. Interestingly upon uniaxial extension, the reflection band shifted towards 


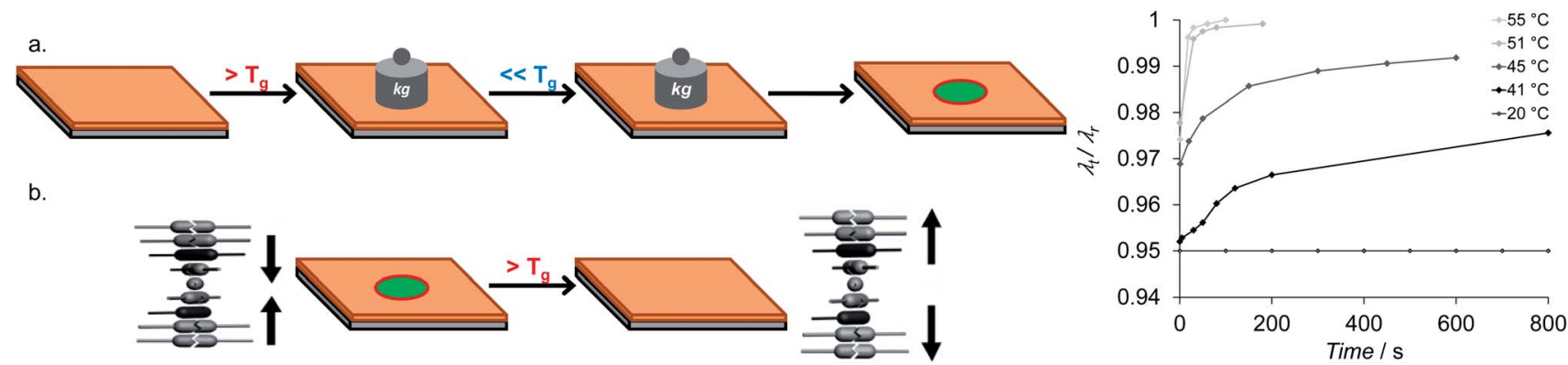

Fig. 14 (a) Fabrication of the irreversible temperature optical sensor by mechanical embossing. A CLC layer is embossed above the glass transition temperature $\left(T_{\mathrm{g}}\right)$ and cooled down below $T_{\mathrm{g}}$ to fix compressive deformation and a blue shift caused by reduction of the helical pitch. (b) Sensing principle of the embossed CLC-layer. The embossed area is preserved below $T_{\mathrm{g}}$ and a permanent red shift is obtained above $T_{\mathrm{g}}$. (C) Time dependent sensor response at different temperatures. At $t=0 \mathrm{~s}$, the sensor had reached the temperature set point, $\lambda_{r}$ is the wavelength of the $\mathrm{SRB}$ after heating the sample to $60^{\circ} \mathrm{C}$ leading to recovery of the original SRB band. Adapted from Davies et al. ${ }^{30} \mathrm{Copyright} \odot 2013 \mathrm{WILEY}$-VCH Verlag GmbH \& Co. KGaA, Weinheim.

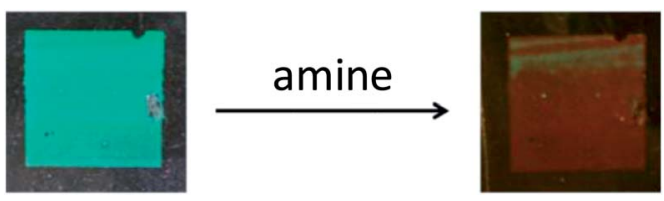

Fig. 15 Photographs of the green reflecting CLC film before and after exposure to spoiling codfish. Adapted from Stumpel et al. ${ }^{31}$ Copyright (c) 2014 WILEY-VCH Verlag GmbH \& Co. KGaA, Weinheim.

lower wavelengths and a $40 \mathrm{~nm}$ shift was recorded for $13 \%$ of strain resulting in a green reflection band (Fig. 16). This wavelength shift was attributed to a decrease in the cholesteric helix's pitch which was induced by the change in thickness of the CLC coating as a result of increasing strain. Repeated loading showed a time dependent optical response of the sensor fitting with the strain of the substrate giving real time information on the deformation.

\section{Conclusions and outlook}

Sensors based on cholesteric liquid crystals can be considered as $1 \mathrm{D}$ photonic materials that are responsive to a wide range of
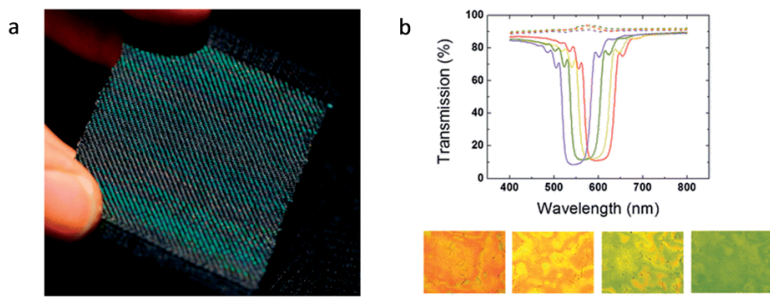

Fig. 16 (a) CLC coated PA6 woven into textile. Reprinted (adapted) with permission of O. T. Picot, et al. ${ }^{32}$ Copyright (2013) American Chemical Society; (b) transmission spectra of a polyamide/CLC bilayer film for different extensions: 0\% (red), 2.6\% (yellow), 7.7\% (green), and $13 \%$ (violet) and microscopy pictures in reflection mode with corresponding change in color. Reproduced from Picot et al..$^{33}$ with permission from The Royal Society of Chemistry. stimuli. They can respond in a real-time or time integrating manner, as can be concluded from the examples discussed. CLC materials can easily be fabricated by using relatively low cost commercially available liquid crystal compounds and chiral dopants. No complex techniques are required to prepare the photonic band gap materials since the structure is assembled by the self-organization of the molecules.

Furthermore, these optical sensors are not sensitive to photobleaching, as seen for sensors based on reactive dyes, since the principle is based on a change of the selective reflection of light caused by the periodic structure. The prepared photonic materials can respond to various analytes by different mechanisms, resulting in a shift of the selective reflection band (SRB).

For small molecule CLC sensors, the mechanism of the change in SRB can be based on responsive chiral dopants. Both blue- and red-shifts have been demonstrated depending on the mechanism. In other small molecule CLC sensors, analyte molecules can penetrate into the cholesteric superstructure, leading to a swelling causing a strong red shift or ultimately disruption of the helical pitch.

Sensors based on CLC polymer network films appear to be mechanically more stable than the low molecular weight systems. CLC polymer films are generally prepared from liquid crystalline molecules consisting of (meth)acrylate groups. After aligning the CLCs, the cholesteric superstructure can be frozen by photo-polymerization. Mechanisms are based on swelling or loss of order of the cholesteric superstructure by the penetration or reaction with analyte molecules. Another mechanism is the change of the cholesteric superstructure by mechanical forces in for instance the time-temperature sensor and the strain sensor discussed.

Various (potential) applications of the chiral nematic sensors have been proposed in articles. For example the use of these materials as optical sensors in the temperature-controlled supply chain of the food and pharmaceutical industries has been proposed. The common temperature range here is 2 to 8 ${ }^{\circ} \mathrm{C}$ but the specific (time integrated) temperature tolerances depend on the product. In the food industry, additional product specific environmental parameters such as carbon dioxide, 
oxygen, amines, humidity and others also play an important role. Other applications mentioned are in the field of anticounterfeiting. For example to detect counterfeited alcoholic beverages containing for instance the toxic methanol. To measure contaminations or chemical species in water, solvent vapour and strain have also been proposed as possible applications.

All the applications mentioned above are still far from being usable in daily life situations. Often the selectivity, sensitivity, and signal changes do not meet the requirements set for the specific applications. Most sensors suffer from cross-selectivity and/or need co-analytes to work properly. For example, organic vapour sensors appeared to be sensitive to fluctuations in temperature and humidity of the environment, and the oxygen sensor only works in the presence of an organic base like triethylamine. Furthermore, sensors do not appear to be sensitive enough for detecting small quantities of the analytes.

Generally, the most sensitive and selective sensors reported so far are based on low molecular weight CLCs in which a responsive chiral dopant is used. However, these samples have a liquid-like nature which hampers their application in for example food packaging. A solution could be to embed the CLC in a polymer binder to create a polymer dispersed liquid crystal in which the good response of low weight systems can be combined with the processability of polymers.

Finally the CLC sensors can be regarded as responsive pigments which could also be interesting for other areas such as architecture, building and textile applications. Recently we have shown that is possible to spray CLC on polyamide fibres and fabricate woven textiles (Fig. 16). ${ }^{33}$ In such applications selectivity and sensitivity are probably less critical parameters.

Overall this work shows that chiral nematic liquid crystals can be used as one dimensional photonic materials that act as optical sensors, but for real applications a number of challenging problems has to be resolved first.

\section{Acknowledgements}

The authors would like to acknowledge the discussions and contributions with all of their former and current colleagues. Their names are given in the references cited. A special word of thanks is expressed to Dick Broer and Rint Sijbesma for many inspiring discussions and collaborations. The research at Eindhoven University of Technology has been supported by the Dutch Polymer Institute (DPI), the Royal Netherlands Academy of Science (KNAW), the Netherlands Organization for Scientific Research (NWO) and the European Young Investigators Awards (EURYI).

\section{Notes and references}

1 J. Ge and Y. Yin, Angew. Chem., Int. Ed., 2011, 50, 1492-1522. 2 (a) H.-S. Kitzerow and C. Bahr, Chirality in Liquid Crystals, Springer-Verlag, New York, 2001; (b) D. J. Broer and I. Heynderickx, Macromolecules, 1990, 23, 2474-2477.

3 T. J. White, M. E. McConney and T. J. Bunning, J. Mater. Chem., 2010, 20, 9832.
4 J. A. Kelly, A. M. Shukaliak, C. C. Y. Cheung, K. E. Shopsowitz, W. Y. Hamad and M. J. Maclachlan, Angew. Chem., Int. Ed., 2013, 52, 8912-8916.

5 M. K. Khan, M. Giese, M. Yu, J. A. Kelly, W. Y. Hamad and M. J. MacLachlan, Angew. Chem., Int. Ed., 2013, 52, 89218924.

6 C. C. Y. Cheung, M. Giese, J. A. Kelly, W. Y. Hamad and M. J. MacLachlan, ACS Macro Lett., 2013, 2, 1016-1020.

7 Y. P. Zhang, V. P. Chodavarapu, A. G. Kirk and M. P. Andrews, Sens. Actuators, B, 2013, 176, 692-697.

8 I. B. Burgess, M. Lončar and J. Aizenberg, J. Mater. Chem. C, 2013, 1, 6075.

9 (a) R. J. Carlton, J. T. Hunter, D. S. Miller, R. Abbasi, P. C. Mushenheim, L. N. Tan and N. L. Abbott, Liq. Cryst. Rev., 2013, 1, 29-51; (b) B. Troia, A. Paolicelli, F. De Leonardis, V. Passaro and M. N. Vittorio, Advances in Photonic Crystals, ed. V. Passaro and M. N. Vittorio, Intech, 2013, pp. 241-295.

10 (a) S. J. Woltman, G. D. Jay and G. P. Crawford, Nat. Mater., 2007, 6, 929-938; (b) H. Xu, P. Wu, C. Zhu, A. Elbaza and Z. Z. Gu, J. Mater. Chem. C, 2013, 1, 6087-6098.

11 F. L. Dickert, A. Haunschild and P. Hofmann, Fresenius' J. Anal. Chem., 1994, 577-581.

12 D. A. Winterbottom, R. Narayanaswamy and I. M. Raimundo, Sens. Actuators, B, 2003, 90, 52-57.

13 A. Mujahid, H. Stathopulos, P. A. Lieberzeit and F. L. Dickert, Sensors, 2010, 10, 4887-4897.

14 C.-K. Chang, H.-L. Kuo, K.-T. Tang and S.-W. Chiu, Appl. Phys. Lett., 2011, 99, 073504.

15 C.-K. Chang, S.-W. Chiu, H.-L. Kuo and K.-T. Tang, Appl. Phys. Lett., 2012, 100, 043501.

16 Y. Han, K. Pacheco, C. W. M. Bastiaansen, D. J. Broer and R. P. Sijbesma, J. Am. Chem. Soc., 2010, 132, 29612967.

17 A. Saha, Y. Tanaka, Y. Han, C. M. W. Bastiaansen, D. J. Broer and R. P. Sijbesma, Chem. Commun., 2012, 48, 4579-4581.

18 X. Wang, C. Drew, S.-H. Lee, K. J. Senecal, J. Kumar and L. a. Samuelson, Nano Lett., 2002, 2, 1273-1275.

19 F. Özogul and Y. Özogul, Food Chem., 2006, 99, 574-578.

20 P. V. Shibaev, K. Schaumburg and V. Plaksin, Chem. Mater., 2002, 14, 959-961.

21 P. V. Shibaev, J. Madsen and A. Z. Genack, Chem. Mater., 2004, 16, 1397-1399.

22 P. V. Shibaev, D. Chiappetta, R. L. Sanford, P. PalffyMuhoray, M. Moreira, W. Cao and M. M. Green, Macromolecules, 2006, 39, 3986-3992.

23 P. V. Shibaev, R. L. Sanford, D. Chiappetta and P. Rivera, Mol. Cryst. Liq. Cryst., 2007, 479, 161.

24 F. Chen, J. Guo, O. Jin and J. Wei, Chin. J. Polym. Sci., 2013, 31, 630-640.

25 F. Chen, J. Guo, Z. Qu and J. Wei, J. Mater. Chem., 2011, 21, 8574.

26 C.-K. Chang, C. W. M. Bastiaansen, D. J. Broer and H.-L. Kuo, Macromolecules, 2012, 45, 4550-4555.

27 C.-K. Chang, C. M. W. Bastiaansen, D. J. Broer and H.-L. Kuo, Adv. Funct. Mater., 2012, 22, 2855-2859. 
28 V. Stroganov, A. Ryabchun, A. Bobrovsky and V. Shibaev, Macromol. Rapid Commun., 2012, 33, 1875-1881.

29 N. Herzer, H. Guneysu, D. J. D. Davies, D. Yildirim, A. R. Vaccaro, D. J. Broer, C. W. M. Bastiaansen and A. P. H. J. Schenning, J. Am. Chem. Soc., 2012, 134, 7608-7611.

30 D. J. D. Davies, A. R. Vaccaro, S. M. Morris, N. Herzer, A. P. H. J. Schenning and C. W. M. Bastiaansen, Adv. Funct. Mater., 2013, 23, 2723-2727.
31 J. E. Stumpel, C. Wouters, N. Herzer, J. Ziegler, D. J. Broer, C. W. M. Bastiaansen and A. P. H. J. Schenning, Adv. Opt. Mater., 2014, 2, 459.

32 O. T. Picot, M. Dai, E. Billoti, D. J. Broer, T. Peijs and C. W. M. Bastiaansen, RSC Adv., 2013, 3, 18794.

33 O. T. Picot, M. Dai, D. J. Broer, T. Peijs and C. W. M. Bastiaansen, ACS Appl. Mater. Interfaces, 2013, 5, 7117-7121. 